\title{
Development of Next Generation Sequencing Protocols for dsRNA Viruses
}

\author{
Sushila Maan ${ }^{1}$, Deepika Chaudhary ${ }^{2}$, Nitish Bansal ${ }^{2}$, \\ Bhanita Devi ${ }^{2}$ and Narender S. Maan ${ }^{2}$ \\ ${ }^{1}$ Department of Animal Biotechnology, ${ }^{2}$ College of Veterinary Sciences, LalaLajpat Rai \\ University of Veterinary and Animal Sciences, Hisar, Haryana, India \\ *Corresponding author
}

\section{Keywords}

dsRNA, cDNA

synthesis,

Tagmented DNA,

Denaturation

Article Info

Accepted:

10 January 2019

Available Online:

10 February 2019
Next generations sequencing (NGS) is a very important tool to identify pathogens in different forms i.e. viruses, bacteria etc. This helpful in decode life mysteries, detect pathogens and improve life qualities. Next generation sequencing/Massively parallel sequencing it allows millions of sequencing reactions to be carried out in parallel due to this accuracy and sequencing rate is high as compare to other sequencing methods. In this communication we tried to develop a protocol for dsRNA viruses for MIseq platform.

\section{Introduction}

Next-generation sequencing methodologies is now changing the way that we understand viruses, particularly in the areas of genome sequencing, evolution, ecology, discovery and transcriptomics. These technologies have opened further applications in virology including routine diagnostic work and new understanding of the interaction between viral and host transcriptomes. Although this opens an exciting era of viral exploration yet we expect new challenges to understand the role of newly discovered viral diversity in both disease and health (Radford et al., 2012, Marston et al., 2013).

In virology NGS methodology offers many benefits like no prior information or knowledge of virus genome is required 
Schuster (2008). Furthermore, NGS is even useful in situation where low number of viral load is present. For execution the most commonly used platform is Illumina. We have recently developed a NGS protocol for dsRNA viruses using MiSeq illumina platform which is described in this report.

\section{Protocols}

\section{Viral RNA isolation}

The viral dsRNA will be extracted from various clinical samples tissue/cell culture isolates/blood/swabs by using different protocols e.g. using Trizol reagents (Attoui et $a l ., 2000)$ or using various RNA isolation kits available from various companies e.g., RNeasy plus universal mini kit (Qiagen). Samples after trizol extraction are treated either with DNase I $(0.5 \mathrm{U} / \mu \mathrm{l}$; TaKaRa Bio Inc., Otsu, Japan) or with S1 nuclease (27 $\mathrm{U} / \mu \mathrm{l}$; TaKaRa Bio Inc.) at $23^{\circ} \mathrm{C}$ for $15 \mathrm{~min}$ as per method described by Shimada et al., (2015).

This extracted RNA will be further used as template for downstream reactions for sequencing. The steps are following:

\section{cDNA synthesis}

\section{First Strand RT}

(For low RNA concentrations samples such as swab, use double concentrations)

\section{Mix I:}

Random hexamer $(50 \mu \mathrm{M}): 1 \mu 1$

RNA: $2 \mu 1$ (50-300ng or cell culture) or $11 \mu 1$ (serum, plasma, CSF)

Water: Up to $12 \mu 1$ total

5 min @ $75^{\circ} \mathrm{C}$, then put on ice

\section{Mix II}

\begin{tabular}{|l|c|}
\hline 5X Buffer & $4 \mu \mathrm{l}$ \\
\hline $0.1 \mathrm{M}$ DTT & $1 \mu \mathrm{l}$ \\
\hline 25mM dNTPs & $1 \mu \mathrm{l}$ \\
\hline RNaseOUT & $1 \mu \mathrm{l}$ \\
\hline $\begin{array}{l}\text { SuperScript III RT } \\
(200 \text { units/ } \mu \mathrm{l})\end{array}$ & $1 \mu \mathrm{l}$ \\
\hline
\end{tabular}

Add Mix II to Mix I

Total volume $20 \mu 1$

Prepare Mix II beforehand or while sample incubates at $75^{\circ} \mathrm{C}$; add Mix II right after Mix I is in the ice bath; mix briefly, quick spin, and incubate at $25^{\circ} \mathrm{C}$ for $10 \mathrm{~min}, 50^{\circ} \mathrm{C}$ for $60 \mathrm{~min}$, $85^{\circ} \mathrm{C}$ for $5 \mathrm{~min}$

\section{RNase H}

Add RNase $\mathrm{H}=1 \mu \mathrm{l}$; Incubate at $37^{\circ} \mathrm{C}$ for 20 $\min , 75^{\circ} \mathrm{C}$ for $15 \mathrm{~min}$

Second Strand synthesis (work inside clean PCR hood)

\begin{tabular}{|l|l|}
\hline cDNA & $21 \mu \mathrm{l}$ \\
\hline 10X NEBuffer 2 & $10 \mu \mathrm{l}$ \\
\hline 25mM dNTPs & $2 \mu \mathrm{l}$ \\
\hline exo- Klenow fragment 5 unit/ul & $4 \mu \mathrm{l}$ \\
\hline Random hexamers (600ng/ul) & $1 \mu \mathrm{l}$ \\
\hline Nuclease-free water & $62 \mu \mathrm{l}$ \\
\hline
\end{tabular}

45 min @ 37 $7^{\circ} \mathrm{C}$

Quantify samples with Qubit 2.0, fluorometer. If the sample is less than $0.05 \mathrm{ng} / \mu \mathrm{l}$, do not use it for library prep.).

\section{Purification of cDNA samples using AM PureXP magnetic beads}

1. Take $90 \mu$ lof cDNA sample, to it add 162 $\mu l$ beads, and mix for two minutes on VWR shaker. Incubate plates for 5 mins at room temperature. 
2. Put this plate on magnetic stand for two minutes at room temperature. Discard the supernatant.

3. While plate on magnetic stand, wash the plates with $80 \%$ ethanol for 30 sec., without disturbing the beads. Discard ethanol and proceed to second wash step.

4. While plate on magnetic stand, wash the plates with $80 \%$ ethanol for $30 \mathrm{sec}$, without disturbing the beads.

5. Discard ethanol and incubate the plate at room temperature for 15 minutes. Make sure that ethanol is completely evaporated.

6. Now add $30 \mu \mathrm{l}$ of elution buffer/NFW to plate and afterwards remove the plate form the magnetic stand. Seal the plate and keep it on shaker for 02 minutes.

7. Again put plate on magnetic stand, transfer whole of the $(30 \mu \mathrm{l})$ elute to the PCR plate.

*Quantify samples with Qubit 2.0, fluorometer. If the sample is less than 0.05 $\mathrm{ng} / \mu 1$, do not use it for library prep.)

\section{cDNA library preparation}

\section{Nextera XT DNA Library Prep Kit}

(https://www.illumina.com/products/bytype/sequencing-kits/library-prep-

kits/nextera-xt-dna.html)

Library preparation is a very important step in NGS based sequencing. This has 5 sub steps. First

Tagment Genomic DNA, Amplify the Libraries, Cleanup of this, normalize these libraries and finally pool it.

\section{Tagment Genomic DNA}

This step uses the Nexteratransposome to tagment gDNA, which is a process that fragments DNA and then tags the DNA with adapter sequences in a single step.

\section{Procedure}

1. Tagment DNA Buffer (TD) $(10 \mu \mathrm{l})$ and normalized gDNA $(5 \mu \mathrm{l})$ are added in new Hard-Shell skirted PCR plate. Add $5 \mu \mathrm{l}$ Amplicon Tagment Mix (ATM) to each well. Pipette to mix.

2. Centrifuge at $280 \times \mathrm{g}$ at $20^{\circ} \mathrm{C}$ for 1 minute.

3. Place on the preprogrammed thermal cycler and run the tagmentation program $\left(55^{\circ} \mathrm{c}\right.$ for 5 minutes, Hold at $\left.10^{\circ} \mathrm{c}\right)$. When the sample reaches $10^{\circ} \mathrm{C}$, immediately proceed to step 5 because the transposome is still active.

4. Add $5 \mu$ INeutralize Tagment Buffer (NT) to each well. Pipette to mix. Centrifuge at $280 \times \mathrm{g}$ at $20^{\circ} \mathrm{C}$ for 1 minute. Incubate at room temperature for 5 minutes.

\section{Amplify Libraries}

This step amplifies the tagmented DNA using a limited-cycle PCR program. PCR adds the Index 1 (i7), Index 2 (i5), and full adapter sequences to the tagmented DNA from the previous step. The index primers and Nextera PCR master mix are added directly to the 25 $\mu \mathrm{l}$ of tagmented gDNA from the previous step. The adapters and sequences are required for cluster formation. Use the full amount of recommended input DNA and the specified number of PCR cycles, which helps ensure high-quality sequencing results. When planning the index scheme for libraries, use the same Index 1 (i7) in each column of the PCR plate. This scheme allows use of a multichannel pipette to transfer indexes from the tubes to the plate.

\section{Procedure}

1. [24 libraries] Arrange the index primers in the TruSeq Index Plate Fixture as follows. Arrange Index 1 (i7) adapters in columns 1-6 of the TruSeq Index Plate Fixture. Arrange Index 2 (i5) adapter in rows A-D 
of the TruSeq Index Plate Fixture.

2. [96 libraries] Arrange the index primers in the TruSeq Index Plate Fixture as follows. Arrange Index 1 (i7) adapters in columns 1-12 of the TruSeq Index Plate Fixture. Arrange Index 2 (i5) adapter in rows A-H of the TruSeq Index Plate Fixture.

3. Using a multichannel pipette, add $5 \mu \mathrm{l}$ of each Index 1 (i7) adapter down each column. Replace the cap on each i7 adapter tube with a new orange cap.

4. Using a multichannel pipette, add $5 \mu \mathrm{l}$ of each Index 2 (i5) adapter across each row. Replace the cap on each i5 adapter tube with a new white cap.

5. Add $15 \mu \mathrm{l}$ NPM to each well containing index adapters. Pipette to mix.

6. Centrifuge at $280 \times \mathrm{g}$ at $20^{\circ} \mathrm{C}$ for 1 minute.

7. Place on the preprogrammed thermal cycler $\left(72^{\circ} \mathrm{c}\right.$ for 3 minutes, $95^{\circ} \mathrm{c}$ for 30 seconds, 12 cycles of: $95^{\circ} \mathrm{c}$ for 10 seconds, $55^{\circ} \mathrm{c}$ for 30 seconds, $72^{\circ} \mathrm{c}$ for 30 seconds, $72^{\circ} \mathrm{C}$ for 5 minutes, Hold at $\left.10^{\circ} \mathrm{c}\right)$.

8. If you are stopping, seal the plate and store at $2^{\circ} \mathrm{C}$ to $8^{\circ} \mathrm{C}$ for up to 2 days. Alternatively, leave on the thermal cycler overnight.

\section{Clean up libraries}

This step uses AMPure XP beads to purify the library DNA and remove short library fragments.

\section{Procedure}

1. Centrifuge at $280 \times \mathrm{g}$ at $20^{\circ} \mathrm{C}$ for 1 minute.

2. Transfer $50 \mu \mathrm{l}$ PCR product from each well of the PCR plate to corresponding wells of a new midi plate.

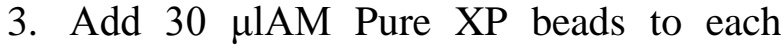
well. Shake at $1800 \mathrm{rpm}$ for 2 minutes. Incubate at room temperature for 5 minutes.

4. Place on a magnetic stand and wait until the liquid is clear ( 2 minutes). Remove and discard all supernatant from each well.

5. Wash two times as follows.

a Add $200 \mu \mathrm{l}$ fresh $80 \%$ EtOH to each well. $b$ Incubate on the magnetic stand for 30 seconds.

c Remove and discard all supernatant from each well.

6. Using a $20 \mu \mathrm{l}$ pipette, remove residual $80 \% \mathrm{EtOH}$ from each well. Air-dry on the magnetic stand for 15 minutes.

7. Remove from the magnetic stand. Add $52.5 \mu \mathrm{l}$ RSB to each well. Shake at 1800 rpm for 2 minutes. Incubate at room temperature for 2 minutes.

8. Place on a magnetic stand and wait until the liquid is clear ( 2 minutes). Transfer $50 \mu \mathrm{l}$ supernatant from the midi plate to a new Hard-Shell PCR plate. If you are stopping, seal the plate and store at $-25^{\circ} \mathrm{C}$ to $-15^{\circ} \mathrm{C}$ for up to 7 days. Quantify library with fragment analyzer and Qubit 2.0, fluorometer.

\section{Normalization of libraries by denaturation of libraries}

MiSeq Reagent Kit v3 = 4 nM libraryResults in a 6-20 pM loading concentration.

MiSeq Reagent Kit v2 $=\mathbf{4}$ nM libraryResults in a 6-20 pM loading concentration.

nM library-Results in a 6-10 pM loading concentration.

\section{Denature a 4 nMlibrary}

1. Combine the following volumes in a microcentrifuge tube. $4 \mathrm{nM}$ library $(5 \mu \mathrm{l})$ and $0.2 \mathrm{~N} \mathrm{NaOH}$ freshly prepared $(5 \mu \mathrm{l})$, 
Vortex briefly and then centrifuge at 280 $\times \mathrm{g}$ for 1 minute.

2. Incubate for 5 minutes at room temperature.

3. Add $990 \mu \mathrm{l}$ prechilled HT1 to the tube containing denatured library. The result is $1 \mathrm{ml}$ of a $20 \mathrm{pM}$ denatured library.

\section{Denature a 2 nM library}

1. Combine the following volumes in a microcentrifuge tube. $2 \mathrm{nM}$ library $(5 \mu \mathrm{l})$ and $0.2 \mathrm{~N} \mathrm{NaOH}(5 \mu \mathrm{l})$, Vortex briefly and then centrifuge at $280 \times \mathrm{g}$ for 1 minute.

2. Incubate for 5 minutes at room temperature.

3. Add $990 \mu 1$ prechilled HT1 to the tube containing denatured library. The result is $1 \mathrm{ml}$ of a $10 \mathrm{pM}$ denatured library.

\section{Dilute denatured $20 \mathrm{pM}$ library}

1. Dilute to the desired concentration using the following volumes.

\begin{tabular}{|l|l|l|l|l|l|l|}
\hline Concentration & $6 \mathrm{pM}$ & $8 \mathrm{pM}$ & $10 \mathrm{pM}$ & $12 \mathrm{pM}$ & $15 \mathrm{pM}$ & $20 \mathrm{pM}$ \\
\hline $20 \mathrm{pM}$ library & $180 \mu \mathrm{l}$ & $240 \mu \mathrm{l}$ & $300 \mu \mathrm{l}$ & $360 \mu \mathrm{l}$ & $450 \mu \mathrm{l}$ & $600 \mu \mathrm{l}$ \\
\hline $\begin{array}{l}\text { Prechilled } \\
\text { HT1 }\end{array}$ & $420 \mu \mathrm{l}$ & $360 \mu \mathrm{l}$ & $300 \mu \mathrm{l}$ & $240 \mu \mathrm{l}$ & $150 \mu \mathrm{l}$ & $0 \mu \mathrm{l}$ \\
\hline
\end{tabular}

2. Invert to mix and then pulse centrifuge.

\section{Dilute PhiX to 4 nM}

Combine the following volumes in a microcentrifuge tube.10 nMPhiX library $(2 \mu \mathrm{l}), \quad 10$ mMTris-Cl, $\mathrm{pH} 8.5$ with $0.1 \%$ Tween $20(3 \mu \mathrm{l})$

\section{Denature PhiX Control}

Combine the following volumes in a microcentrifuge tube. $4 \mathrm{nMPhiX}$ library $(5 \mu \mathrm{l})$, $0.2 \mathrm{~N} \mathrm{NaOH}(5 \mu \mathrm{l})$, Vortex briefly to mix. Centrifuge at $280 \times \mathrm{g}$ for 1 minute. Incubate at room temperature for 5 minutes.

\section{Dilute denatured PhiX to $20 \mathrm{pM}$}

Add prechilled HT1 to the denatured PhiX library. Denatured PhiX library $(10 \mu \mathrm{l})$, Prechilled HT1 $(990 \mu \mathrm{l})$. The result is $1 \mathrm{ml}$ of a 20 pMPhiX library.

Combine library and PhiXcontrol as shown in below table. This put on ice until you are ready to load it onto the reagent cartridge.

\begin{tabular}{|l|l|l|}
\hline & $\begin{array}{l}\text { Most } \\
\text { Libraries } \\
(1 \% \text { Spike- } \\
\text { In) }\end{array}$ & $\begin{array}{l}\text { Low- } \\
\text { Diversity } \\
\text { Libraries } \\
(\geq \quad 5 \% \\
\text { Spike-In) }\end{array}$ \\
\hline $\begin{array}{l}\text { Denatured and } \\
\text { diluted PhiX }\end{array}$ & $6 \mu 1$ & $30 \mu 1$ \\
\hline $\begin{array}{l}\text { Denatured and } \\
\text { diluted library } \\
\text { (from protocol A } \\
\text { or protocol B) }\end{array}$ & $594 \mu 1$ & $570 \mu 1$ \\
\hline
\end{tabular}

\section{MiSeq workflow}

MiSeq reagent kit microv2, MiSeq reagent kit v3

1. Prepare the prefilled reagent cartridge for use.

2. Load the library mix onto the reagent cartridge in the designated reservoir.

3. From the software interface, select Sequence to start the run setup steps. Wash and thoroughly dry the flow cell. Load the flow cell.

4. Load the PR2 bottle and make sure that the waste bottle is empty. Load the 
reagent cartridge.

5. Review run parameters and pre-run check results. Select Start Run.

6. Monitor your run from the MCS interface or from another computer using Sequencing Analysis Viewer (SAV).

7. Perform a post-run wash.

\section{Cluster generation}

During cluster generation, single DNA molecules are bound to the surface of the flow cell, and then bridge-amplified to form clusters.

\section{Sequencing}

Following cluster generation, clusters are imaged using LED and filter combinations specific to each of the 4 fluorescently labelled dideoxynucleotides. After imaging of a tile is complete, the flow cell is moved into place to expose the next tile.

The process is repeated for each cycle of sequencing. Following image analysis, the software performs base calling, filtering, and quality scoring.

\section{Analysis}

When the run is complete, the MiSeq Reporter analysis software launches automatically to perform secondary analysis, which includes alignment and variant calling.

\section{Acknowledgments}

This work was supported by the Biotechnology and Biological Sciences Research Council (BBSRC) and Department of Biotechnology, Government of India (DBT, GOI) (Grant no. BT/IN/IndoUK/FADH/46/SM/2013) to SM.

\section{References}

Attoui H, Billoir F, Cantaloube JF, Biagini P, de Micco $P$ and de Lamballerie $X$, (2000) Strategies for the sequence determination of viral dsRNA genomes. J Virol Methods 89:147158.

Marston, DA, McElhinney LM, Ellis RJ, Horton DL, Wise EL, Leech SL, David D, Lamballerie, X and Fooks AR (2013) Next generation sequencing of viral RNA genomes. BMC Genomics 14:444.

Radford AD, Chapman D, Dixon L, Chantrey J, Darby AC, Hall N (2012) Application of next-generation sequencing technologies in virology. $\mathrm{J}$ Gen Virol93:1853-1868.

Schuster SC (2008) Next-generation sequencing transforms today's biology. Nat Methods. 5:16-18.

Shimada S, Nagai M, Moriyama H, Fukuhara T, Koyama S, Omatsu T, Furuya T, Shiraj J (2015)Use of S1 nuclease in deep sequencing for detection of double-stranded RNA viruses. J Vet Med Sci 77: 1163-1166.

\section{How to cite this article:}

Sushila Maan, Deepika Chaudhary, Nitish Bansal, Bhanita Devi and Narender S. Maan. 2019. Development of Next Generation Sequencing Protocols for dsRNA Viruses. Int.J.Curr.Microbiol.App.Sci. 8(02): 1062-1067. doi: https://doi.org/10.20546/ijcmas.2019.802.125 